\title{
Mapping and Ablation of Frequent Post-Infarction Premature Ventricular Complexes
}

\author{
JEAN-FRANCOIS SARRAZIN, M.D., ERIC GOOD, D.O., MICHAEL KUHNE, M.D., \\ HAKAN ORAL, M.D., FRANK PELOSI, JR, M.D., AMAN CHUGH, M.D., \\ KRIT JONGNARANGSIN, M.D., THOMAS CRAWFORD, M.D., \\ MATTHEW EBINGER, D.O., FRED MORADY, M.D., and FRANK BOGUN, M.D.
}

From the Department of Cardiology, University of Michigan Cardiovascular Center, Ann Arbor, Michigan, USA

\begin{abstract}
Mapping of Post-Infarction PVCs. Introduction: Premature ventricular complexes (PVCs) occur frequently in patients with heart disease. The sites of origin of PVCs in patients with prior myocardial infarction and the response to catheter ablation have not been systematically assessed.

Methods and Results: In 28 consecutive patients ( 24 men, age $60 \pm 10$, ejection fraction [EF] $0.37 \pm 0.14$ ) with remote myocardial infarction referred for catheter ablation of symptomatic refractory PVCs, the PVCs were mapped by activation mapping or pace mapping using an irrigated-tip catheter in conjunction with an electroanatomic mapping system. The site of origin (SOO) was classified as being within low-voltage (scar) tissue (amplitude $\leq 1.5 \mathrm{mV}$ ) or tissue with preserved voltage $(>1.5 \mathrm{mV}$ ). The SOO was confined to endocardial scar tissue in $24 / 28$ patients $(86 \%)$. The SOO was outside of scar in 3 patients and could not be identified in 1 patient. At the SOO, local endocardial activation preceded the PVC by $46 \pm 19 \mathrm{~ms}$, and the electrogram amplitude during sinus rhythm was $0.48 \pm 0.34 \mathrm{mV}$. The PVCs were effectively ablated in $25 / 28$ patients $(89 \%)$, resulting in a decrease in PVC burden on a 24-hour Holter monitor from $15.6 \pm 12.3 \%$ to $2.4 \pm 4.2 \%(P<0.001)$. The SOO most often was confined to scar tissue located in the left ventricular septum and the papillary muscles.

Conclusion: Similar to post-infarction ventricular tachycardia, PVCs after remote myocardial infarction most often originate within scar tissue. Catheter ablation of these PVCs has a high-success rate. (J Cardiovasc Electrophysiol, Vol. 21, pp. 1002-1008, September 2010)
\end{abstract}

catheter ablation, myocardial infarction, premature ventricular complexes, electroanatomic mapping, antiarrhythmic drugs

\section{Introduction}

Catheter ablation of frequent premature ventricular complexes (PVCs) has a high-success rate in patients without structural heart disease. ${ }^{1}$ Only a few case reports have described mapping and ablation of post-infarction PVCs. ${ }^{2,3}$ In a small series of post-infarction patients, the impact of radiofrequency ablation of frequent PVCs on left ventricular ejection fraction (LVEF) was described. ${ }^{4}$ The purpose of the study was to describe mapping and ablation of postinfarction PVCs in a larger series of consecutive patients. Twenty-one patients were included in previously published reports. ${ }^{3,4}$

Dr. Oral reports participation on research projects supported by St. Jude Medical and Boston Scientific. He serves as a consultant or on the advisory board of Medtronic. Other authors: No disclosures.

Address for correspondence: Frank Bogun, M.D., Assistant Professor of Medicine, Division of Cardiology, CVC Cardiovascular Medicine, 1500 East Medical Center Drive SPC 5853, Ann Arbor, MI 48109-5853, USA. Fax: +734-936-4027; E-mail: fbogun@med.umich.edu

Manuscript received 18 March 2009; Revised manuscript received 23 February 2010; Accepted for publication 2 March 2010.

doi: 10.1111/j.1540-8167.2010.01771.x
Methods

\section{Characteristics of Subjects (Table 1)}

Twenty-eight consecutive patients (24 men, mean age $60 \pm 10$ years, mean LVEF $0.37 \pm 0.14$ ) referred for catheter ablation of symptomatic post-infarction PVCs, refractory to medical therapy (including $\beta$-blocker and/or antiarrhythmic agents), were analyzed. All patients had remote myocardial infarctions (MI; mean infarct age $9 \pm 6$ years). It was anterior in 7 patients, inferior in 18 patients, septal in 1 patient, and lateral in 2 patients. All patients had an ischemic work-up prior to the ablation. Fifteen patients were treated with antiarrhythmic drugs before the procedure, either for ventricular tachycardia (VT) $(\mathrm{n}=12)$ or refractory PVCs $(\mathrm{n}=3)$. Five patients were on 2 antiarrhythmic drugs simultaneously. Thirteen patients had an implantable cardioverter defibrillator (ICD) implanted before the mapping procedure.

\section{PVC Burden}

The PVC burden (i.e., the percentage of all QRS complexes that were PVCs) was measured with a 24-hour Holter monitor performed before and 3 months after the ablation procedure, if possible with a 12-lead recording system. Eight patients had only one PVC morphology and 20 patients had pleomorphic PVCs. In patients with pleomorphic PVCs, the predominant PVC was a PVC accounting for $>50 \%$ of the occurring PVCs based on the Holter analysis. 


\section{TABLE 1}

Patient Characteristics

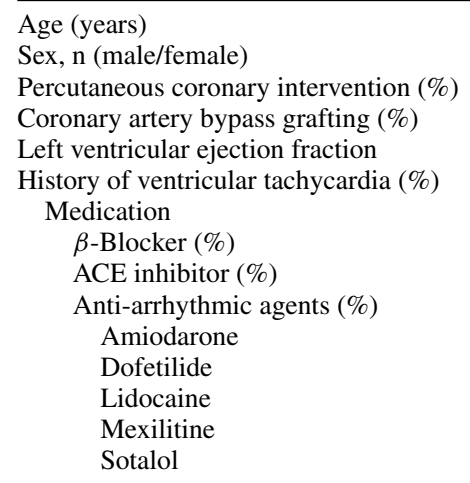

A 12-lead electrocardiogram (ECG) with the predominant PVC morphology was obtained prior to the intervention or at the beginning of the procedure but prior to catheter insertion.

\section{Evaluation of Left Ventricular Ejection Fraction}

All patients had a baseline echocardiogram performed before the ablation. The LVEF was calculated by the Simpson formula when 2 consecutive sinus beats were present, using the second sinus beat for analysis of the LVEF to avoid post-extrasystolic potentiation of left ventricular function. A repeat echocardiogram was performed early post-ablation in all patients (mean $83 \pm 96$ days; median 67 days) and at a later time post-ablation in 13 patients (mean $10.4 \pm$ 7.9 months).

\section{Electrophysiology Study}

The institutional review committee at the University of Michigan approved the protocol. The electrophysiology procedures were performed in the fasting state after informed consent was obtained. A quadripolar electrode catheter was advanced via a femoral vein to the right ventricular apex. Programmed ventricular stimulation was performed with up to 4 extrastimuli at 3 basic drive cycle lengths to determine the inducibility of sustained VT. ${ }^{5}$ VTs were targeted for ablation only if they remained inducible after the PVCs were ablated. Left ventricular access was obtained with a retrograde aortic approach. A bolus of 5,000 units of heparin was administered followed by an infusion of 1,000 units/hour to maintain an activated clotting time of approximately 300 seconds. Bipolar electrograms were filtered at 50-500 Hz. ECG leads and intracardiac electrograms were displayed on an oscilloscope. The recordings were stored on optical disc (EP Medical Systems, New Berlin, NY, USA).

\section{Mapping and Ablation}

An electroanatomical mapping system (CARTO, Biosense Webster, Diamond Bar, CA, USA) was used to create a left ventricular voltage map during sinus rhythm. In patients with pleomorphic PVCs, only the predominant PVCs were mapped and ablated during the procedure. PVCs occurring frequently (bigeminal to quadrigeminal patterns) were mapped with activation mapping. If the ectopy remained sporadic (i.e., not in repetitive pattern), pace-mapping was

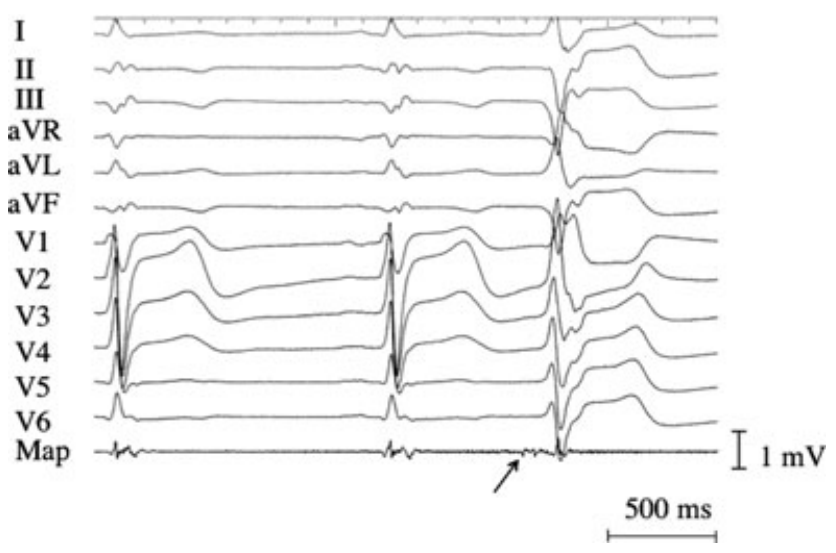

Figure 1. Twelve-lead ECG tracing showing 2 sinus rhythm beats followed by a premature ventricular complex $(P V C)$. The recording from the mapping catheter (MAP) indicates the earliest endocardial activation time at the effective ablation site of the PVC (arrow).

performed at a pacing cycle length that matched the coupling interval of the spontaneous ventricular ectopy. ${ }^{6}$ Mapping and ablation was performed with a $3.5-\mathrm{mm}$ irrigated-tip catheter (ThermoCool, Biosense Webster). The earliest activation time of the PVCs was recorded (Fig. 1). The local bipolar amplitude at the effective ablation site was recorded during sinus rhythm. The presence of isolated potentials, defined as potentials separated from the ventricular electrogram by an isoelectric segment of more than $20 \mathrm{~ms}$, was also noted. Bipolar pacing was performed with the distal electrode pair of the catheter (1-mm inter-electrode space from the ring electrode).

The sites of origin (SOO) of the PVCs were classified as being within a region of low voltage (low voltage was defined as a bipolar voltage amplitude $\leq 1.5 \mathrm{mV}$; this was referred in the manuscript as scar) or a region of preserved voltage $(>1.5 \mathrm{mV})$. In addition, the region of low voltage was further separated either in dense scar $(<0.5 \mathrm{mV})$ or in border zone $(0.5-1.5 \mathrm{mV})$. The anatomic SOO of each targeted PVC was documented in conjunction with the activation time and the electrogram amplitude during sinus rhythm at the effective ablation site.

The power of the radiofrequency energy applications was titrated upwards to a maximum of $50 \mathrm{~W}$ to achieve an impedance drop of $10 \Omega$. In the event of pleomorphic PVCs (present in 20 patients), the predominant PVC morphologies were targeted. Programmed ventricular stimulation was repeated at the end of the procedure.

\section{Follow-Up}

Antiarrhythmic drug therapy was discontinued if the ablation procedure was effective. In patients who also had VT, antiarrhythmic medication that was ineffective prior to the ablation procedure was continued post procedure at the same dosage. This included amiodarone in 3 patients. No new medications were started. The patients were seen in an arrhythmia clinic or ICD clinic every 3-6 months during follow-up.

A 24-hour Holter monitor was repeated at 3-6 months after the ablation procedure to reassess the PVC burden. A successful outcome was defined as $\geq 80 \%$ reduction in PVC burden. 
TABLE 2

PVC Characteristics for Each Patient

\begin{tabular}{|c|c|c|c|c|c|c|c|}
\hline Patients & $\begin{array}{l}\text { PVC Pre- } \\
\text { RFA }(\%)\end{array}$ & $\begin{array}{c}\text { \# PVC (\# PVCs } \\
\text { Targeted) }\end{array}$ & SOO & SOO Scar & Success & $\begin{array}{l}\text { PVC Post- } \\
\text { RFA }(\%)\end{array}$ & $\begin{array}{l}\text { Follow-up } \\
\text { (Months) }\end{array}$ \\
\hline 1 & 43.5 & $1(1)$ & Basal LVOT & $S$ & $\mathrm{Y}$ & 3.2 & 3.0 \\
\hline 2 & 38.3 & $13(5)$ & $\mathrm{PAP}$ anterior $+\mathrm{CVS} \times 3+$ septum & $\mathrm{S} \times 1$ & $\mathrm{Y}$ & 16.7 & 3.5 \\
\hline 3 & 33.3 & $6(2)$ & Left aortic cusp + basal LVOT & $\mathrm{B} \times 1$ & $\mathrm{Y}$ & 1.0 & 3.5 \\
\hline 4 & 32.3 & $1(1)$ & PAP anterior & $\mathrm{S}$ & $\mathrm{Y}$ & 0.7 & 29.5 \\
\hline 5 & 32.0 & $1(1)$ & Mitral annulus & B & $\mathrm{Y}$ & 0.5 & 19.0 \\
\hline 6 & 30.0 & $8(3)$ & PAP anterior $\times 2+$ para-Hisian & $\mathrm{S} \times 2$ & $\mathrm{Y}$ & 0.4 & 12.5 \\
\hline 7 & 27.0 & $6(1)$ & Basal LVOT & B & $\mathrm{Y}$ & 0.0 & 4.0 \\
\hline 8 & 24.3 & $5(2)$ & Inferior + MA & $\mathrm{B} \times 1$ & $\mathrm{Y}$ & 0.2 & 2.0 \\
\hline 9 & 18.0 & $1(1)$ & Antero-lateral basal & B & $\mathrm{Y}$ & 0.0 & 28.0 \\
\hline 10 & 15.9 & $4(2)$ & Antero-basal $\times 2$ & $\mathrm{~S} \times 2$ & $\mathrm{~N}$ & 3.0 & 23.5 \\
\hline 11 & 15.5 & $6(2)$ & Inferior $\times 2$ & $\mathrm{~B} \times 2$ & $\mathrm{Y}$ & 11.4 & 3.0 \\
\hline 12 & 14.2 & $1(1)$ & PAP posterior & $\mathrm{S}$ & $\mathrm{Y}$ & 0.1 & 24.0 \\
\hline 13 & 14.1 & $2(1)$ & Basal LVOT & $\mathrm{S}$ & $\mathrm{Y}$ & 0.3 & 9.0 \\
\hline 14 & 13.9 & $2(1)$ & Septum & $\mathrm{S}$ & $\mathrm{Y}$ & 0.2 & 23.0 \\
\hline 15 & 13.3 & $1(1)$ & PAP posterior & B & $\mathrm{Y}$ & 11.8 & 3.0 \\
\hline 16 & 11.7 & $10(5)$ & Septum $\times 5$ & $\mathrm{~B} \times 3 \mathrm{~S} \times 2$ & $\mathrm{Y}$ & 3.3 & 11.0 \\
\hline 17 & 11.0 & $2(1)$ & CVS & $\mathrm{N}$ & $\mathrm{Y}$ & 2.0 & 2.5 \\
\hline 18 & 10.3 & $2(2)$ & $\mathrm{PAP}$ anterior $\times 2$ & $\mathrm{~S} \times 2$ & $\mathrm{Y}$ & 1.2 & 7.0 \\
\hline 19 & 7.0 & $1(1)$ & Left posterior fascicle & $\mathrm{S}$ & $\mathrm{Y}$ & 1.0 & 17.0 \\
\hline 20 & 6.0 & $8(1)$ & CVS & $\mathrm{N}$ & $\mathrm{Y}$ & 0.0 & 13.0 \\
\hline 21 & 6.0 & $5(1)$ & Unable to locate & $\mathrm{N}$ & $\mathrm{N}$ & 6.0 & 28.0 \\
\hline 22 & 5.3 & $5(1)$ & Inferior & $\mathrm{S}$ & $\mathrm{Y}$ & 0.7 & 18.0 \\
\hline 23 & 5.0 & $2(1)$ & Septum & B & $\mathrm{Y}$ & 0.2 & 20.0 \\
\hline 24 & 5.0 & $5(1)$ & Septum & $\mathrm{S}$ & $\mathrm{Y}$ & 2.0 & 17.0 \\
\hline 25 & 3.0 & $1(1)$ & Intramural & B & $\mathrm{Y}$ & 0.0 & 16.0 \\
\hline 26 & 0.3 & $3(1)$ & Intramural & $\mathrm{N}$ & $\mathrm{N}$ & 0.2 & 16.5 \\
\hline 27 & 0.2 & $2(2)$ & PAP posterior $\times 2$ & $\mathrm{~S} \times 2$ & $\mathrm{Y}$ & 0.0 & 21.5 \\
\hline 28 & 0.2 & $4(1)$ & Inferior & $\mathrm{S}$ & $\mathrm{Y}$ & 0.0 & 8.0 \\
\hline Average or total & 15.6 & $108(44)$ & & 33 & $25(89 \%)$ & 2.4 & 13.8 \\
\hline
\end{tabular}

$\mathrm{B}=$ border zone; $\mathrm{CVS}=$ coronary venous system; LVOT $=$ left ventricular outflow tract; $\mathrm{MA}=$ mitral annulus; $\mathrm{N}=$ no; PAP $=$ papillary muscle; $\mathrm{PVC}=$ premature ventricular contractions; $\mathrm{RFA}=$ radiofrequency ablation; $\mathrm{S}=$ dense scar; $\mathrm{SOO}=$ site of origin; $\mathrm{Y}=$ yes.

\section{Statistical Analysis}

Continuous variables are expressed as the mean \pm 1 standard deviation and were compared using paired Student's $t$ test. A P value of $<0.05$ was considered significant.

\section{Results}

\section{Mapping and Origin of PVCs (Table 2)}

A total of 108 different PVCs were recorded during the ablation procedures, with a mean of 3.9 PVCs/patient (median 2.5 PVCs/patient). Forty-four morphologies of PVCs were mapped (41\%; mean 1.6 PVCs/patient; median 1.0 PVC/patient). The mapped PVCs accounted for $86 \%$ of the total PVC burden in these patients. The average PVC coupling interval was $578 \pm 114 \mathrm{~ms}$. The PVC morphologies were right bundle branch block and superior axis in 13 cases, right bundle branch block and inferior axis in 20, left bundle branch block and superior axis in 4 , and left bundle branch block and inferior axis in 7 .

The SOO of the predominant PVC was identified in 27 of 28 patients $(96 \%)$, by activation mapping in 13 patients, by pace-mapping in 6 patients, and by both techniques in 9 patients. The SOO was within endocardial scar in 24 of 28 patients $(86 \%)$, accounting for 33 of 44 PVCs $(75 \%)$. From these 33 PVCs, 13 (39\%) were located in the border zone and $20(61 \%)$ within the dense scar. In patients with remote inferolateral MI $(\mathrm{n}=19), 28$ PVCs were targeted and 21/28 PVCs (75\%) were located within the inferior scar (inferoseptal $=10$, inferior $=5$, papillary muscle $=4$, and posterolateral $=1)$. The remaining PVCs originated from the epicardium $(n=4)$, the left ventricular outflow tract (LVOT; $\mathrm{n}=1)$, the aortomitral continuity $(\mathrm{n}=1)$, and the aortic cusp $(\mathrm{n}=1)$. One PVC could not be located. In patients with a prior anteroseptal and lateral MI $(\mathrm{n}=9), 16 \mathrm{PVCs}$ were targeted and 12/16 PVCs (75\%) were located within the anterior scar (papillary muscle $=6, \mathrm{LVOT}=3$, and anterior $=2$ ). The remaining PVCs originated from the aortic cusp $(n=2)$ or the conduction system (His bundle $=1$ and left posterior fascicle $=1$ ). The SOO was in a region of preserved voltage in 3 of 28 patients and could not be identified in 1 patient. Overall, the SOO of the mapped PVCs were at left ventricular septum $(\mathrm{n}=12)$, the papillary muscle $(\mathrm{n}=10)$, the LVOT or aortic cusp $(\mathrm{n}=7)$, the epicardium $(\mathrm{n}=5)$, the inferior wall $(\mathrm{n}=5)$, the anterolateral wall $(\mathrm{n}=3)$, and the aortomitral continuity $(\mathrm{n}=1)$. The epicardial SOO was reached from the greater cardiac vein in 3 patients. The anatomical distribution of the SOO is shown in Figure 2, and the different PVC morphologies in Figures 3 and 4.

The SOO was located an average of $19 \pm 16 \mathrm{~mm}$ from the border of left ventricular scar (Fig. 5). At the SOO, local endocardial activation preceded the PVC by an average of $46 \pm 19 \mathrm{~ms}$, and the mean electrogram amplitude during baseline rhythm was $0.48 \pm 0.34 \mathrm{mV}$. Isolated potentials during sinus rhythm were found in 12 patients at the SOO. The mean stimulus-to-QRS interval during pace-mapping was $56 \pm 16 \mathrm{~ms}$. 


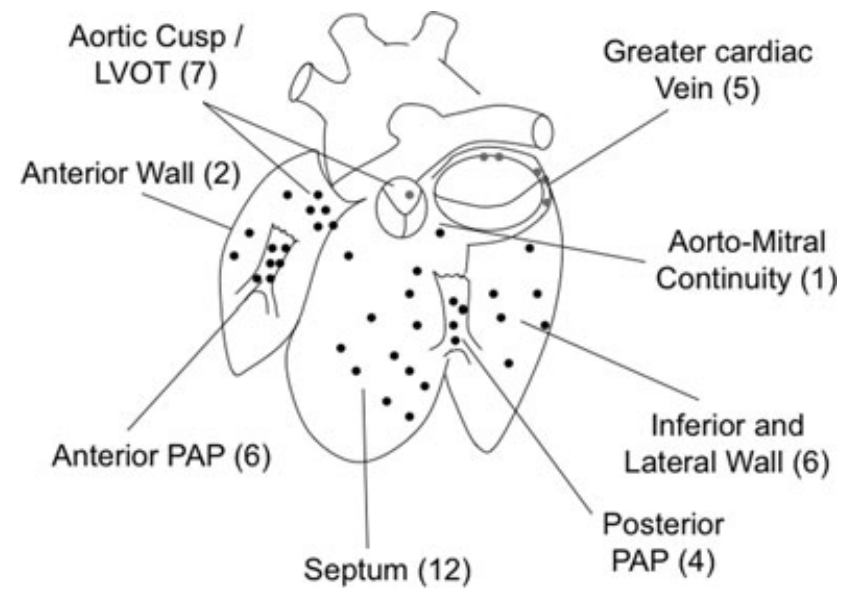

Figure 2. Anatomical distribution of the premature ventricular complex $(P V C)$ sites of origin (SOO). Each black dot represents an endocardial PVC $S O O$ and each gray dot represents an epicardial PVC SOO. PAP = papillary muscle; $L V O T=$ left ventricular outflow tract.

\section{Pleomorphic PVCs}

In 9 of 20 patients with pleomorphic PVCs, two or more morphologies of PVCs were targeted during the same procedure. The maximum number of PVCs targeted during the same procedure was 5 . Overall, $41 \%$ of the total PVCs were targeted. Targeted PVCs originated from the same area in 8 of 9 patients. The average distance between the SOO of the predominant $\mathrm{PVC}$ and the SOO of the other PVCs was $27 \pm 16 \mathrm{~mm}$.

\section{PVC Ablation and Complications}

Forty of 44 targeted PVCs (91\%) were ablated acutely in 25 of 28 patients (89\%). An average of $13 \pm 14$ radiofrequency energy applications was required for ablation of the predominant PVC. The average duration of radiofrequency was $19.7 \pm 15.9$ minutes. The average procedure time was $309 \pm 101$ minutes, with a fluoroscopy time of $60 \pm 24$ minutes.

Fifteen patients (54\%) had a total of 46 inducible sustained VTs. Sixteen of the 46 VTs were reproducibly inducible. After ablation of 16 PVCs, 9 VTs remained inducible and were targeted subsequently.

Two complications occurred. The first was 2:1 atrioventricular block with a new left bundle branch block during ablation near the left posterior fascicle in a patient with preexisting right bundle branch block and prolonged HV interval. This patient already had an ICD and also had interfascicular VT. The second complication was a femoral arteriovenous fistula managed conservatively.

\section{Follow-Up}

No patients died during a mean follow-up period of $13.8 \pm 8.9$ months (median 14.5 months). Repeat Holter monitoring (performed at a mean of $56 \pm 36$ days postablation) demonstrated a significant decrease in the PVC burden from $15.6 \pm 12.3$ to $2.4 \pm 4.2 \%(\mathrm{P}<0.001)$. The success rate at 3-6 months post-PVC ablation was $75 \%$. The PVC burden on repeat Holter monitoring was $2.1 \pm 4.0 \%$ for patients with monomorphic PVC, $2.4 \pm 4.3 \%$ for patients with pleomorphic PVCs, and $4.1 \pm 5.9 \%$ for patients who had 2 or more PVCs targeted during the procedure

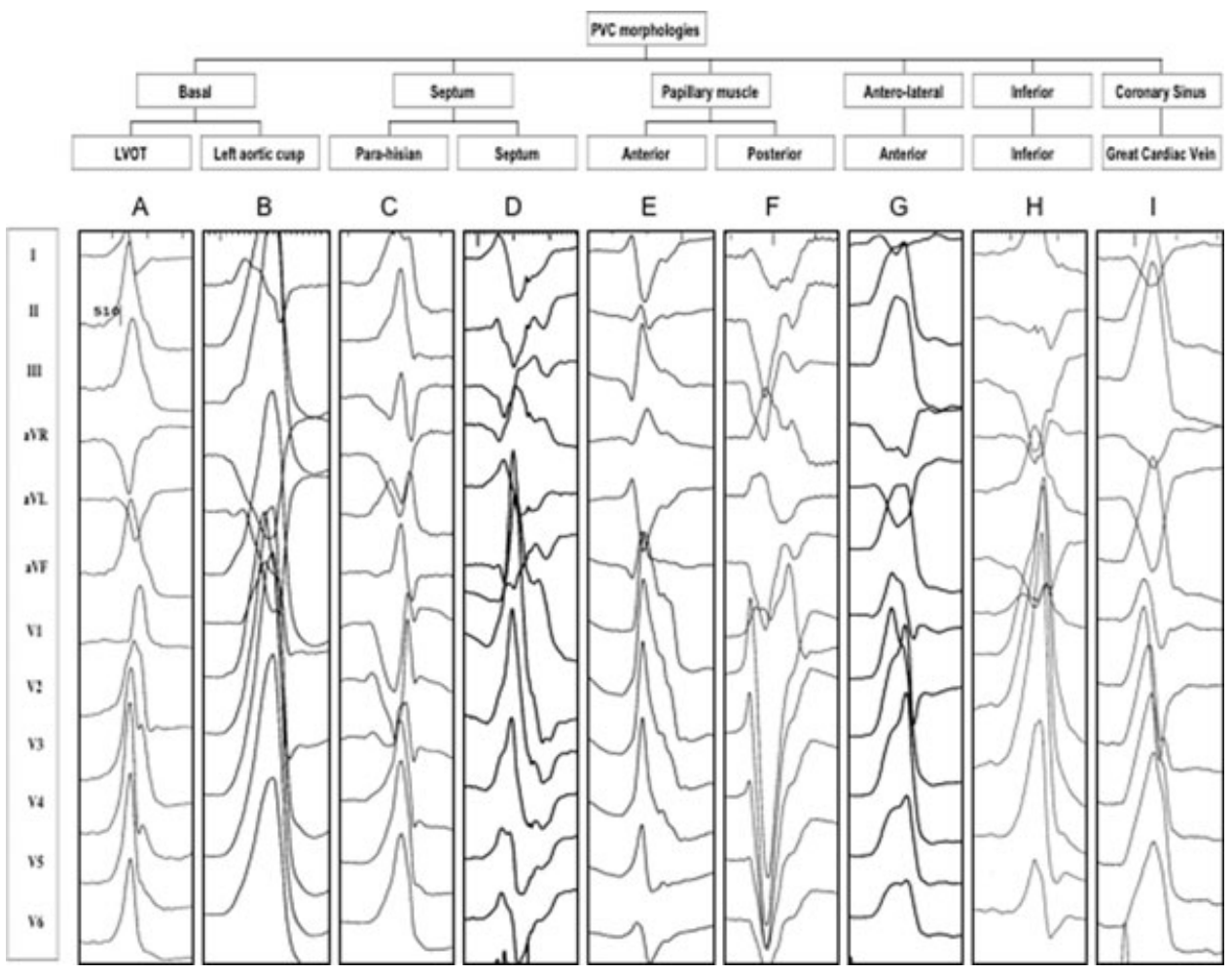

Figure 3. Nine different premature ventricular complex (PVC) morphologies (named A to I) on 12-lead ECG from several patients are incorporated into a flow chart based on their intracardiac location. 
A

C

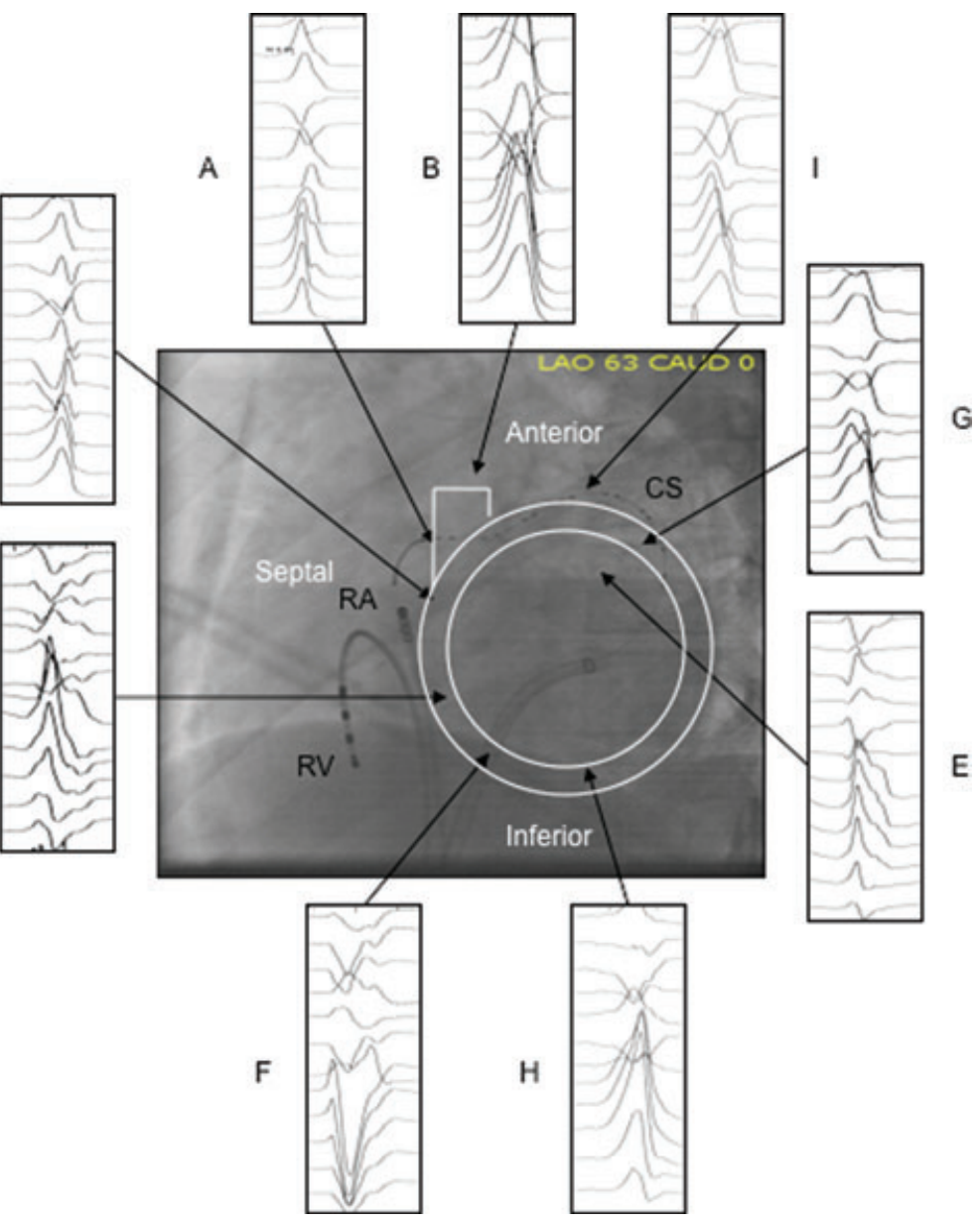

B

B

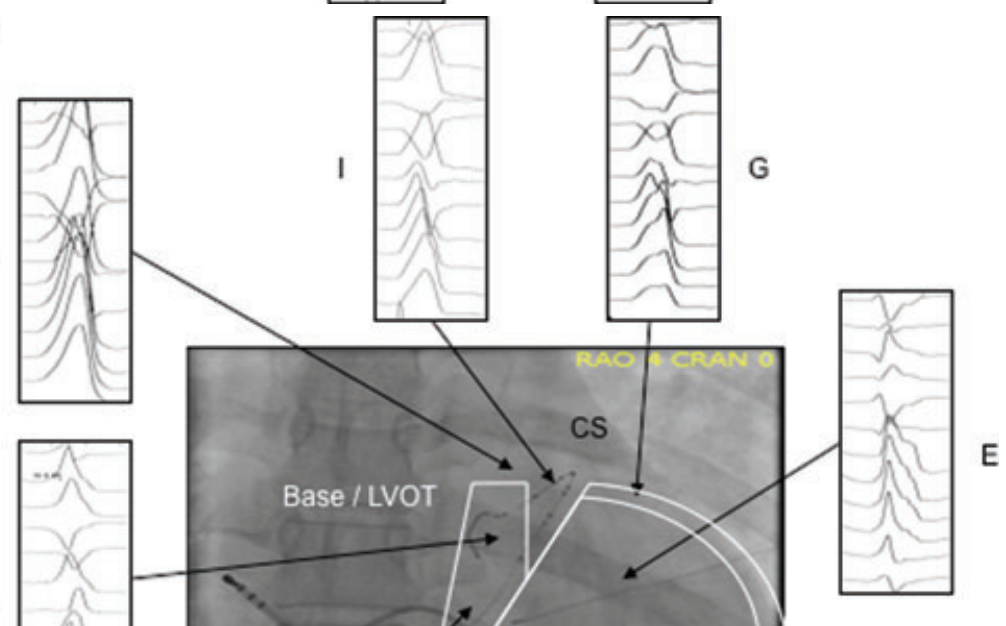

c

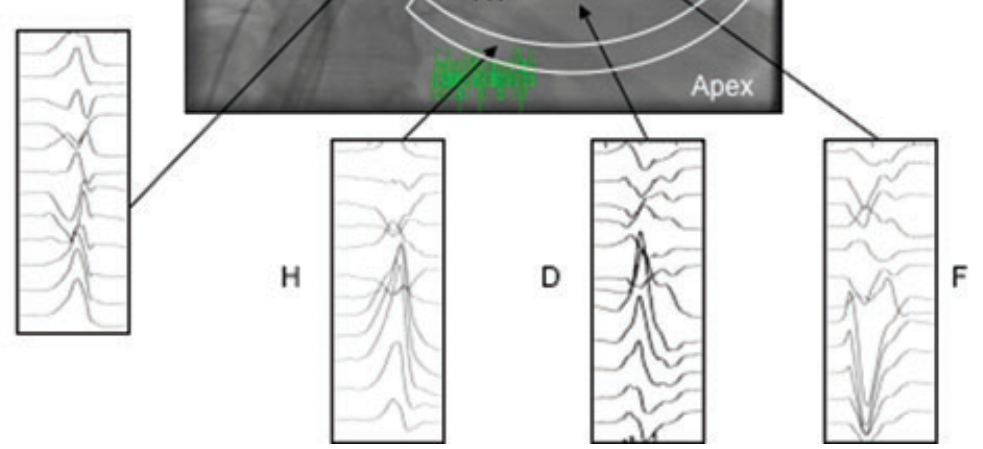

Figure 4. Same premature ventricular complex morphologies (A to I) as Figure 3 with their respective site of origin in relationship to fluoroscopic images: $A=L A O$ view and $B=R A O$ view. $C S=$ multipolar catheter in great cardiac vein; $R A=$ catheter in the right atrium; $R V=$ catheter in the right ventricle. The left ventricular cavity is schematically displayed in red. 


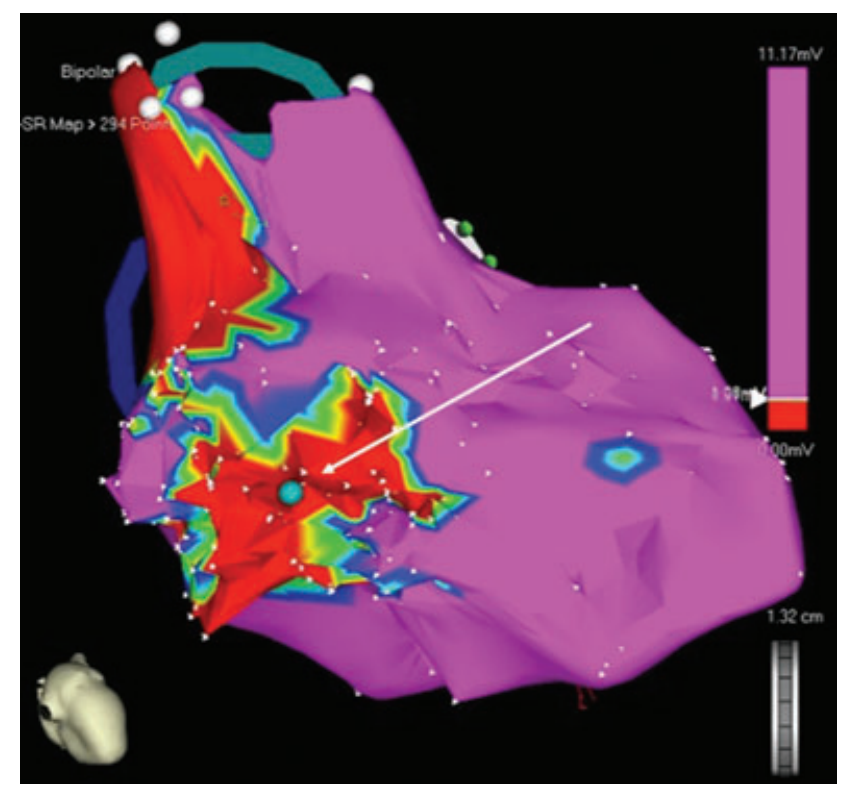

Figure 5. Electroanatomic voltage map of the left ventricle with a septal scar. The premature ventricular complex originated from the center of the scar (blue dot and arrow).

$(\mathrm{P}=\mathrm{ns})$. Ten out of 15 patients with inducible VT had less than $1 \%$ PVC burden and no VT during the follow-up.

There was a small increase in the mean LVEF early post-ablation (from $0.37 \pm 0.14$ to $0.39 \pm 0.13 ; \mathrm{P}=0.2$ ). The difference became significant with longer follow-up $(0.37 \pm 0.14$ prior to ablation to $0.43 \pm 0.15 ; \mathrm{P}=0.03)$.

\section{Discussion}

In the majority of patients with remote MI and frequent PVCs, the PVCs originate at sites of low voltage. Mapping and ablation of post-infarction PVCs can be performed endocardially with a high-success rate. The most common sites of origin are the left ventricular septum and a papillary muscle.

\section{Mapping and Origin of Post-Infarction PVCs}

There are only a few case reports of successful PVC ablation in patients with ischemic heart disease. ${ }^{2,3}$ In this series of consecutive patients with prior MI, PVCs were systematically mapped and ablated. The SOO of most PVCs was in the left ventricular endocardium. Furthermore, in most patients, the effective ablation sites were confined to sites of low voltage corresponding with the infarct location. In several of the patients, the scar tissue gave rise to multiple PVCs that were mapped and ablated. Voltage mapping in these patients was helpful in focusing the mapping procedure to the low-voltage areas, since this area contained the arrhythmogenic substrate in most patients. In a minority of patients, the SOO of the mapped PVCs was at a site of normal voltage, similar to the SOO of idiopathic PVCs.

\section{Similarities with VT Ablation}

PVCs in patients with remote MI have several aspects in common with post-infarction VT. With both types of ar- rhythmias, critical target sites are within areas of low voltage. Isolated potentials are frequently present at effective ablation sites of VT and PVCs. ${ }^{3,7}$ Furthermore, as demonstrated in a prior study, post-infarction PVCs and VT often share critical areas, and mapping and ablation of frequent PVCs can eliminate VT in these patients. ${ }^{3}$

Therefore, it is not unexpected that PVCs in patients with ischemic cardiomyopathy originate mainly from scar tissue in approximately $85 \%$ of patients, similar to patients with ischemic VT. Verma et al. demonstrated that hemodynamically stable ischemic VTs originate mainly from the scar border area. ${ }^{8}$ In our study, PVCs originated near the limit of the dense scar area and the border zone as demonstrated by a mean electrogram amplitude in sinus rhythm of $0.48 \pm 0.34 \mathrm{mV}$. In another study, a human histopathology analysis confirmed the correlation between the voltage map from an electroanatomic map and the scar tissue found on autopsy. ${ }^{9}$ Dense scar correlated with transmural fibrosis, while the border zone had intermediate fibrosis. Low-voltage areas on the endocardium serve as the arrhythmia substrate for most patients with ventricular arrhythmias in the setting of ischemic heart disease. ${ }^{10}$ Adjustment of the voltage color range can be helpful in order to limit the zone of pace-mapping in patients with infrequent PVCs.

\section{Mechanisms of PVC}

Most PVCs originated from scar tissue. Possible mechanisms include reentry, triggered activity, or abnormal automaticity. Because of several features in common with post-infarction $\mathrm{VT},{ }^{3}$ reentry might play a principal role in post-infarction PVCs. High-resolution mapping might be required to prove that reentry is the mechanism of postinfarction PVCs. PVCs that did not originate from scar tissue could be due to triggered activity or abnormal automaticity, similar to idiopathic PVCs.

\section{PVC Ablation and LVEF}

The mean LVEF improved post-PVC ablation, as in a previous publication, ${ }^{4}$ partially explained by overlap in the patient's populations. However, the difference occurred later during follow-up. This may be explained by the larger number of patients with inducible VT, who may have had a higher scar burden.

\section{Limitations}

In patients with pleomorphic PVCs, the SOO was not identified for all PVCs, and it is possible that some of the PVCs originated from sites other than the ones described in this study. Unipolar recordings were not systematically used in this study but they could have helped to identify the site of origin. PVC burden was assessed by 24-hour Holter monitoring before and after the ablation. Because of the short period of recording, it is possible that the PVC burden has been underestimated during the follow-up.

\section{Conclusion}

Similar to post-infarction VT, PVCs in patient with ischemic cardiomyopathy and remote MI most often originate at endocardial sites with low voltage. Catheter ablation can be performed safely and with a high-success rate. 


\section{References}

1. Bogun F, Crawford T, Reich S, Koelling TM, Armstrong W, Good E, Jongnarangsin K, Marine JE, Chugh A, Pelosi F, Oral H, Morady F: Radiofrequency ablation of frequent, idiopathic premature ventricular complexes: Comparison with a control group without intervention. Heart Rhythm 2007;4:863-867.

2. Szumowski L, Sanders P, Walczak F, Hocini M, Jais P, Kepski R, Szufladowicz E, Urbanek P, Derejko P, Bodalski R, Haissaguerre M: Mapping and ablation of polymorphic ventricular tachycardia after myocardial infarction. J Am Coll Cardiol 2004;44:17001706.

3. Bogun F, Crawford T, Chalfoun N, Kuhne M, Sarrazin JF, Wells D, Good E, Jongnarangsin K, Oral H, Chugh A, Pelosi F, Morady F: Relationship of frequent postinfarction premature ventricular complexes to the reentry circuit of scar-related ventricular tachycardia. Heart Rhythm 2008;5:367-374.

4. Sarrazin JF, Labounty T, Kuhne M, Crawford T, Armstrong WF, Desjardins B, Good E, Jongnarangsin K, Chugh A, Oral H, Pelosi F, Morady F, Bogun F: Impact of radiofrequency ablation of frequent postinfarction premature ventricular complexes on left ventricular ejection fraction. Heart Rhythm 2009;6:1543-1549.

5. Hummel D, Strickberger S, Daoud E, Niebauer M, Bakr O, Man K, Williamson B, Morady F: Results and efficency of programmed ventricular stimulation with four extrastimuli compared with one, two, and three extrastimuli. Circulation 1994;90:2827-2823.
6. Goyal R, Harvey M, Daoud EG, Brinkman K, Knight BP, Bahu M, Weiss R, Bogun F, Man KC, Strickberger SA, Morady F: Effect of coupling interval and pacing cycle length on morphology of paced ventricular complexes. Implications for pace mapping. Circulation 1996;94:2843-2849.

7. Bogun F, Good E, Reich S, Elmouchi D, Igic P, Lemola K, Tschopp D, Oral H, Chugh A, Pelosi F, Morady F: Isolated potentials during sinus rhythm and pace-mapping within scars as guides for ablation of postinfarction ventricular tachycardia. J Am Coll Cardiol 2006;47:20132019.

8. Verma A, Marrouche NF, Schweikert RA, Saliba W, Wazni O, Cummings J, Abdul-Karim A, Bhargava M, Burkhardt JD, Kilicaslan F, Martin DO, Natale A: Relationship between successful ablation sites and the scar border zone defined by substrate mapping for ventricular tachycardia post-myocardial infarction. J Cardiovasc Electrophysiol 2005; $16: 465-471$.

9. Deneke T, Muller KM, Lemke B, Lawo T, Calcum B, Helwing M, Mugge A, Grewe PH: Human histopathology of electroanatomic mapping after cooled-tip radiofrequency ablation to treat ventricular tachycardia in remote myocardial infarction. J Cardiovasc Electrophysiol 2005; 16:1246-1251.

10. Marchlinski F, Garcia F, Siadatan A, Sauer W, Beldner S, Zado E, Hsia H, Lin D, Cooper J, Verdino R, Gerstenfeld E, Dixit S, Russo A, Callans D: Ventricular tachycardia/ventricular fibrillation ablation in the setting of ischemic heart disease. J Cardiovasc Electrophysiol 2005;16(Suppl 1):S59-S70. 